\title{
Clinical features and disease course of patients with acute ischaemic stroke just before the Italian index case: Was COVID-19 already there?
}

\author{
Anna M. Pietroboni ${ }^{1,2}\left(\right.$ C) Silvia Lanfranconi ${ }^{1,2} \cdot$ Alessio Novella $^{3} \cdot$ Tiziana Carandini $^{1,2} \cdot$ Andrea Arighi $^{1,2}$. \\ Elena Abati ${ }^{1,2} \cdot$ Roberta Brusa $^{1,2}$. Gianluca Costamagna ${ }^{1,2}$. Giulia Lazzeri ${ }^{1,2}$. Eleonora Mauri ${ }^{1,2} \cdot$ Mattia Pozzato $^{1,2}$. \\ Luca Sacchi $^{1,2}$. Gloria Valcamonica ${ }^{1,2}$. Davide Villa ${ }^{1,2}$. Sara Bonato ${ }^{1,2}$. Giacomo P. Comi ${ }^{1,2} \cdot$ Nereo Bresolin $^{1,2}$. \\ Daniela Galimberti ${ }^{1,2}$. Elio Scarpini ${ }^{1,2} \cdot$ Alessandro Nobili $^{3}$ on behalf of the Neurology-COVID-19 Group
}

Received: 23 December 2020 / Accepted: 4 January 2021 / Published online: 10 February 2021

(c) Società Italiana di Medicina Interna (SIMI) 2021

\begin{abstract}
Since the end of February 2020, Italy has suffered one of the most severe outbreaks of coronavirus disease 2019 (COVID19). However, what happened just before the Italian index case has not yet been investigated. To answer this question, we evaluated the potential impact of COVID-19 on the clinical features of a cohort of neurological inpatients admitted right before the Italian index case, as compared to the same period of the previous year. Demographic, clinical, treatment and laboratory data were extracted from medical records. The data collected included all inpatients who had been admitted to the Neurology and Stroke Units of the Ospedale Maggiore Policlinico, Milan, Italy, from December 15, 2018 to February 20, 2019 and from December 15, 2019 to February 20, 2020. Of the 248 patients, 97 subjects (39.1\%) were admitted for an acute cerebrovascular event: 46 in the 2018/2019 period (mean [SD] age, 72.3 [15.6] years; 22 men [47.8\%]), and 51 in the 2019/2020 interval (mean [SD] age, 72.8 [12.4] years; 24 men [47.1\%]). The number of cryptogenic strokes has increased during the 2019-2020 year, as compared to the previous year (30 [58.8\%] vs. 18 [39.1\%], $p=0.05$ ). These patients had a longer hospitalization (mean [SD] day, 15.7 [10.5] days vs. mean [SD] day, 11.7 [7.2] days, $p=0.03$ ) and more frequent cerebrovascular complications $(9[30.0 \%]$ vs. 2 [11.1\%]), but presented a lower incidence of cardiocerebral risk factors (18 $[60.0 \%]$ vs. 14 [77.8\%]). Right before the Italian index case, an increase in cryptogenic strokes has occurred, possibly due to the concomitant COVID-19.
\end{abstract}

Keywords COVID-19 $\cdot$ Acute ischaemic stroke $\cdot$ Cryptogenic stroke $\cdot$ Cerebrovascular events

\section{Introduction}

Since February 20, 2020, Italy, and in particular its most populous region, Lombardy, has suffered one of the most severe outbreaks of coronavirus disease 2019 (COVID-19), caused by the severe acute respiratory syndrome coronavirus

The members of the Neurology-COVID-19 Group was processed under acknowledgements section.

Anna M. Pietroboni

anna.pietroboni@policlinico.mi.it

1 Fondazione IRCCS Ca' Granda Ospedale Maggiore Policlinico, Via F. Sforza 35, 20122 Milan, Italy

2 University of Milan, Dino Ferrari Center, Milan, Italy

3 Istituto di Ricerche Farmacologiche Mario Negri IRCCS, Milan, Italy
2 (SARS-CoV-2). As of December 10, 2020, in this region, inhabited by about $10,000,000$ people, the cases registered by the authorities were more than 430,000 and the total deaths were about 23,000 [1].

Early molecular epidemiological tracing suggests that COVID-19 spread in Lombardy weeks before the first reported cases of infection, confirming that SARS-CoV-2 virus entered northern Italy as early as January, 2020 [2]. A very recent paper confirmed this finding, describing the earliest evidence of SARS-CoV-2 RNA in a patient in Lombardy in early December, 2019, 3 months before Italy's first reported COVID-19 case [3].

The clinical manifestations of COVID-19 mostly affect the respiratory system, configuring varied clinical pictures ranging from mild upper respiratory tract illness to severe pneumonia with respiratory failure and death $[4,5]$. Asymptomatic infections are reported [6]. More recently, 
the virus-related clinical spectrum has widened, including stroke, venous thromboembolism, pulmonary embolism and cardiac complications [7]. The main pathogenic hypothesis focuses on the severe inflammatory state secondary to the infection that leads to an alteration of hemostasis [7, 8]. Neurological manifestations appear to be quite common, including acute cerebrovascular disease, conscious disturbance, headache and olfactory dysfunctions $[9,10]$.

Based on the assumption that COVID-19 may have already been circulating before we knew it, we turned our attention to the period right before the first index patient, focusing on clinical and laboratory features, as well as treatment and disease course, of a cohort of neurological inpatients. To avoid comparing different epidemiological periods and different clinical protocols, we ended the patients' enrollment one day before Italian first reported COVID-19 case.

\section{Methods}

\section{Study design and participants}

This retrospective cohort study included adult inpatients. Our study enrolled all patients who were hospitalized for neurological diseases from December 15, 2018, to February 20, 2019 (Group 1) and from December 15, 2019, to February 20, 2020 (Group 2) to the Neurology and Stroke Units of the Ospedale Maggiore Policlinico of Milan.

The study was approved by the Local Ethics Committee of the Fondazione IRCCS Ca' Granda Ospedale Maggiore Policlinico (363_2020, approved 05.05.2020).

\section{Data collection}

Demographical, clinical, laboratory and treatment data were extracted from electronic medical records using standardized anonymized data collection forms. All data were extracted by eleven physicians (A.M.P, S.L, E.A, R.B, G.C, G.L, E.M, M.P, L.S, G.V and D.V). Routine blood examinations comprised complete blood count, erythrocyte sedimentation rate, serum biochemical tests, including C-reactive protein, liver and renal function, lactate dehydrogenase, creatine kinase and coagulation profile. Instrumental examinations ranged according to clinical need. For acute cerebrovascular disease, they comprised as needed computed tomography (CT) scan of the brain, brain magnetic resonance imaging (MRI), vessel imaging and cardiovascular examinations, including extra- and intracranial ultrasound and transthoracic and transesophageal echocardiography.

Cryptogenic strokes were identified using the TOAST classification [11] by consensus of two independent observers (A.M.P; S.L).

\section{Statistical analysis}

The patients' characteristics were described using percentages for discrete variables, mean and standard deviations for continuous variables. Differences between two groups were analyzed using $F$ test from univariate ANOVA and Pearson's chi-squared test.

Focusing on subjects with stroke, differences in prevalence of cryptogenic events between groups were evaluated using a logistic regression adjusted for age, gender, presence of cardiocerebrovascular (CCV) risk factors and related home drug therapy.

Statistical significance was set up to 0.05 ; as there are no preliminary data, it is not possible to predict whether the size of the collected sample reached the appropriate statistical power.

\section{Results}

Two hundred forty-eight patients were included. Of these, about $40 \%$ was hospitalized for acute cerebrovascular events: 46 in the 2018/2019 period (Group 1) and 51 in 2019/2020 (Group 2). The fraction of patients admitted to the Stoke Unit was not significantly different in the two period and there were no differences in access to acute phase therapies as intravenous fibrinolysis, endovascular treatment or bridging therapy nor in hemorrhagic complications in relation to acute reperfusion treatments. No significant divergences between Groups 1 and 2 regarding demographical characteristics and comorbidities, including hypertension, diabetes, hypercholesterolemia and coronary heart disease were observed (Table 1A). No significant differences were seen in the diagnostic workup of the two periods, targeted from time to time based on the clinical suspicion.

Group 2 patients had longer duration of hospitalization (mean [SD] day, 15.7 [10.5] days vs mean [SD] day, 11.7 [7.4] days, $p=0.04$ ) and higher incidence of cerebrovascular complications (13 [25.5\%] vs 4 [8.7\%], $p=0.03$ ) (Table 1A), which include new ischaemic events and/or hemorrhagic infarctions. During admission, treatment modifications, such as addition of anticoagulant thromboprophylaxis with low-molecular weight heparin and/or switching antiplatelet drugs, occurred more frequently in Group 2 (46 [90.2\%] vs. 30 [69.8\%], $p=0.008$ ) (Table 1A).

Fever and laboratory reports were similar between the two groups. Fibrinogen, D-dimer and serum ferritin were not assessed, as not part of the haematochemistry routine during pre-COVID-19 era.

The frequency of cryptogenic strokes was increased during the 2019/2020 period (30 [58.8\%] vs. 18 [39.1\%], $p=0.05$ ) (Fig. 1a). Group 2 patients had a longer hospitalization (mean [SD] day, 15.7 [10.5] days vs. mean [SD] day, 
Table 1 (A)Main characteristics of patients with stroke included in the study, (B) Logistic model for predicting of cryptogenetic stroke

\begin{tabular}{|c|c|c|c|}
\hline Variables, $N(\%)$ & $\begin{array}{l}\text { Years 2019-2020 } \\
N=51\end{array}$ & $\begin{array}{l}\text { Years 2018-2019 } \\
N=46\end{array}$ & $p$ value \\
\hline \multicolumn{4}{|l|}{ (A) } \\
\hline Gender (female) & $27(52.9)$ & $24(52.2)$ & 0.93 \\
\hline Age, mean (SD) & $72.8(12.4)$ & $72.3(15.7)$ & 0.86 \\
\hline Age $>65$ years & $38(74.5)$ & $34(74.0)$ & 0.95 \\
\hline Cardiocerebrovascular risk factors & $35(68.6)$ & $35(76.1)$ & 0.41 \\
\hline Comorbidites (at least 1 ) & $43(84.3)$ & $40(87.0)$ & 0.71 \\
\hline \multicolumn{3}{|l|}{ Type of stroke (TOAST Classification) } & 0.33 \\
\hline 1. Large-artery atherosclerosis & $5(9.8)$ & $4(9.7)$ & \\
\hline 2. Cardioembolism & $8(15.7)$ & $13(28.3)$ & \\
\hline 3. Small-vessel occlusion & $3(5.9)$ & $5(10.9)$ & \\
\hline 4. Stroke of other determined etiology & $5(9.8)$ & $6(13.0)$ & \\
\hline 5. Stroke of undetermined etiology & $30(58.8)$ & $18(39.1)$ & \\
\hline \multicolumn{3}{|l|}{ TOAST classification } & 0.05 \\
\hline $1-4$ & \multicolumn{2}{|l|}{$21(41.2)$} & \\
\hline 5 & $30(58.8)$ & $18(39.1)$ & \\
\hline Stroke complications & $20(39.2)$ & $10(21.7)$ & 0.06 \\
\hline Cerebrovascular complications & $13(25.5)$ & $4(8.7)$ & $\mathbf{0 . 0 3}$ \\
\hline Other complications & $20(39.2)$ & $19(41.3)$ & 0.83 \\
\hline Cardiac complications & $3(5.8)$ & $7(15.2)$ & 0.13 \\
\hline Infective complications & $14(27.5)$ & $11(23.9)$ & 0.69 \\
\hline Pulmonary infections & $11(21.6)$ & $5(10.87)$ & 0.16 \\
\hline Respiratory complications & $11(21.6)$ & $6(13.0)$ & 0.27 \\
\hline \multicolumn{3}{|l|}{ Drug therapies at home, mean (SD) } & 0.15 \\
\hline Antiplatelet agents & $21(41.2)$ & $22(48.9)$ & \\
\hline Anticoagulants & $2(3.9)$ & $7(15.5)$ & \\
\hline Dual antiplatelet therapy & $1(2.0)$ & 0 & \\
\hline Antiplatelets and anticoagulants & $1(2.0)$ & 0 & \\
\hline Changes in therapy during hospitalization & & & 0.008 \\
\hline Nothing & $5(9.8)$ & $15(33.3)$ & \\
\hline Horizontal shift & $23(45.1)$ & $17(37.8)$ & \\
\hline Anticoagulant shift & $9(17.7)$ & $8(17.8)$ & \\
\hline Dual antiplatelets shift & $4(7.9)$ & $5(11.1)$ & \\
\hline Heparin prophylaxis & $3(5.9)$ & 0 & \\
\hline Heparin + antiplatelets & $7(13.7)$ & 0 & \\
\hline Days of hospitalization, mean (SD) & $15.7(10.5)$ & $11.7(7.4)$ & 0.04 \\
\hline Deaths & $1(2.0)$ & $2(4.4)$ & 0.50 \\
\hline Variables & Odc & ce interval) & $p$ value \\
\hline (B) & & & \\
\hline Group (years 2019-2020 vs. years 2018-2019) & 2.33 & & 0.052 \\
\hline Age & 1.0 & & 0.74 \\
\hline Gender (female vs. male) & 1.12 & & 0.79 \\
\hline $\mathrm{CCV}$ risk factors & 0.4 & & 0.13 \\
\hline Home drugs therapy (yes vs. no) & 2.18 & & 0.11 \\
\hline
\end{tabular}

Bold value indicates statistically significant $(p<0.05)$

11.7 [7.2] days, $p=0.03)$, more frequent cerebrovascular complications (9 $[30.0 \%]$ vs. $2[11.1 \%]$ ), although presenting a lower incidence of CCV risk factors (18 [60.0\%] vs. 14
[77.8\%]) (Fig. 1b-d). In these patients, a certain increase in respiratory complications, including pulmonary infection (6 [20.0\%] vs. 2 [11.1\%]), was observed (Fig. 1e). 
A

Years 2019-2020

5. stroke of undetermined etiology

4. stroke of other determined etiology

3. small-vessel occlusion

2. cardioembolism

1. large-artery atherosclerosis

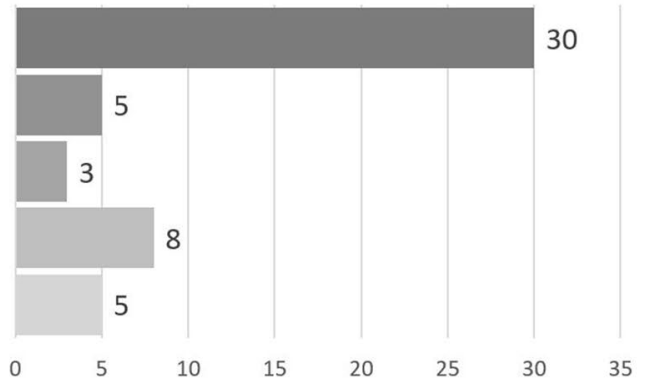

Years 2018-2019

5. stroke of undetermined etiology

4. stroke of other determined etiology

3. small-vessel occlusion

2. cardioembolism

1. large-artery atherosclerosis

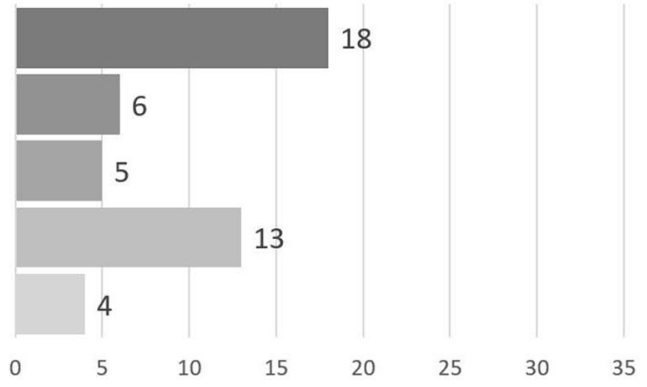

B

Hospitalization

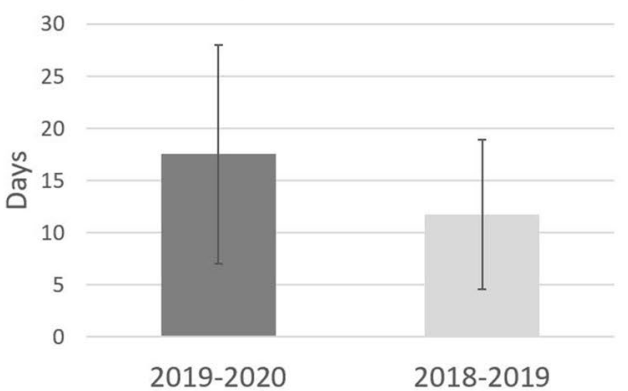

D

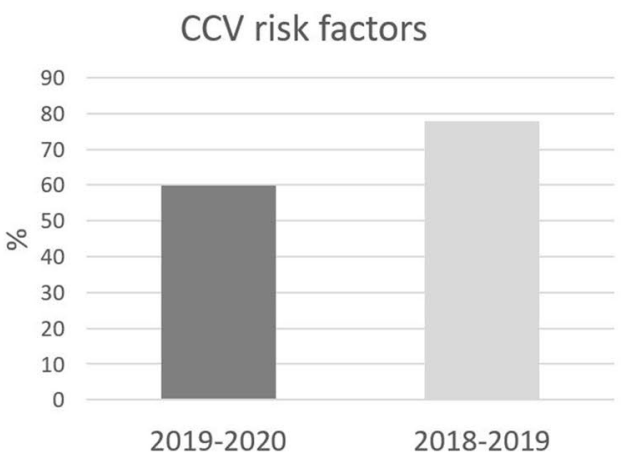

C Cerebrovascular complications

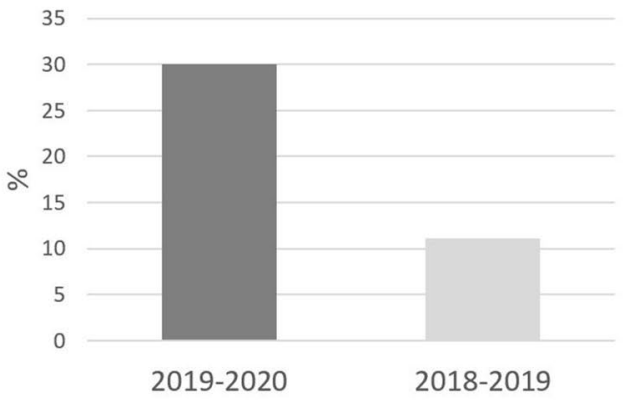

E

Respiratory complications

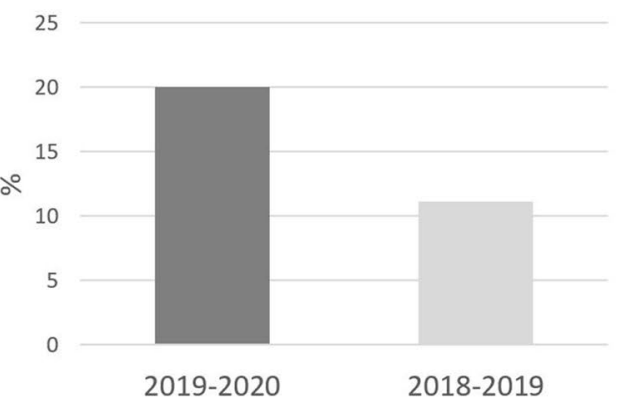


4Fig. 1 a Bar graph showing the percentage of stroke subtypes in the year 2019/2020 vs. 2018/2019. $p=0.05$.b Column graph showing days of hospitalization (mean, SD) in the year 2019-2020 vs. 2018/2019. $p=$ 0.03.c Column graph showing the percentage of cerebrovascular complications in the year $2019 / 2020$ vs. 2018/2019.d Column graph showing the percentage of cardio-cerebrovascular (CCV) risk factors in the year2019/2020 vs. 2018/2019.e Colum graph showing the percentage of respiratory complications in the year $2019 / 2020$ vs. 20

Although the statistical significance was not achieved, logistic models showed a relationship between cryptogenic strokes and period of hospitalization: patients of Group 2 had a twofold increased risk of having a cryptogenic stroke as compared to those of Group 1 (Table 1B).

Forty-three out of 51 total strokes in the 2019/2020 interval were then contacted on the phone. The calls occurred in May, 2020. Only 11 patients had undergone swab for COVID-19 or COVID-19 antibody test. To avoid later infections, only tests carried out within one month of hospitalization were considered. Seven were positive and four were negative. All cryptogenic strokes tested resulted positive (6 of 6), while none of the negatives was a cryptogenic stroke.

\section{Discussion}

In December 2019, January and February, 2020 a significant increase in the number of cryptogenic strokes for no apparent reason could be observed. When compared with the data available in the literature, in which cryptogenic strokes account for $30-40 \%$ of ischaemic strokes [12], it is noteworthy that, in the current year, cryptogenic subtypes were about $60 \%$ of all strokes. COVID-19 had not revealed itself yet, at least expressly, changing the clinical routine and the diagnostic protocols of our wards, which did not differ from that of the 2018/2019 period. In our opinion, the strength of this study lies precisely in comparing two periods in which diagnostic path were similar and not yet derailed due to the advent of COVID-19. Since our wards continued with the diagnostic routine only until the first half of March 2020, we decided not to include in our study the patients hospitalized from February 21,2020 , exactly for not to compare a pre-COVID with a COVID period. That was especially the case with our Stroke Unit, which continued working as a "Level Stroke Unit" on the Stroke Network until mid-March, 2020 only.

Comparing Groups 1 and 2, patients admitted this year were neither older nor younger and did not have a higher number of comorbidities. Instead, they presented lower incidence of $\mathrm{CCV}$ risk factors, but unexpectedly had more complicated hospitalizations. No laboratory tests seem to explain these data. The presence, although still unknown, of COVID-19 in the territory of Milan could hold the answer, even though the true relationship between COVID-19 and cryptogenic stroke incidence remains to be determined.
By now, the current evidence suggests that COVID-19 may predispose patients to thrombotic disease due to excessive inflammation, platelet activation, endothelial dysfunction and stasis [7]. Recent reports described microvascular endothelial injury in postmortem evaluation in multiple organs, consistent with a thrombotic microangiopathy $[13,14]$. This evidence may explain the frequent reports of cerebrovascular complications, also in patients with lower CCV risk factors.

To better understand the link between cerebrovascular accidents and COVID-19, the crucial point seems to focus on the asymptomatic patients. Asymptomatic infections refer to the positive detection of nucleic acid of SARS-CoV-2 by reverse transcriptase polymerase chain reaction in samples of patients who have no typical respiratory symptoms or signs [15]. Therefore, the possible explanations of the increased frequency of cryptogenic strokes, we have experienced seem to be: first, endothelial dysfunction, involving the thrombi formation in the cerebral vessels, may develop even in asymptomatic patients; second, our patients were in the early stages of their systemic viral illness that would soon become clinically evident.

To confirm this last hypothesis, we contacted all 2019/2020 stroke cases. Some patients experienced a cough, fever and difficulty breathing shortly after being discharged, but unfortunately, only 11 had undergone swab for COVID19 or COVID-19 antibody test. Interestingly, all cryptogenic strokes tested resulted positive, while none of the negatives was a cryptogenic stroke. This low number of tests should not be surprising, because most of the patients were first hospitalized and then discharged before we started to consider COVID-19 infections. Recalling all patients to test them for antibody titre was an option. Currently, our understanding of antibody responses following infection with SARS-CoV-2 is limited and more importantly the duration of responses remains unclear. In the light of this, we decided not to test our patients as a year later seemed too distant a time. Moreover, we were worried about not introducing confounding variables, such as post-observation infections, taking into account, the second wave of COVID-19 which has struck Lombardy since early October, 2020.

Limitations of the current study include the retrospective, single centre cohort design, and the small sample size, which could be the reason why the statistical threshold for differences could be not reached. Moreover, the data related to swab for COVID-19 or COVID-19 antibody test was available only for a few patients.

\section{Conclusions}

Right before the Italian index case, an increase in cryptogenic strokes has occurred. Growing evidence suggests that the higher incidence of cryptogenic stroke in asymptomatic 
COVID-19 patients or in the early stages of their systemic illness could be associated with a transient hypercoagulable state, leading to thrombi formation in the cerebral vessels. Replication in a larger cohort of patients is needed to confirm these data.

Acknowledgements Neurology-COVID-19 group members: Federica Arienti: ${ }^{1}$ Fondazione IRCCS Ca' Granda Ospedale Maggiore Polilclinico, Via F. Sforza 35, 20122 Milan, Italy, ${ }^{2}$ University of Milan, Dino Ferrari Center, Milan, Italy; Stefania Corti: ${ }^{1}$ Fondazione IRCCS Ca' Granda Ospedale Maggiore Polilclinico, Via F. Sforza 35, 20122 Milan, Italy, ${ }^{2}$ University of Milan, Dino Ferrari Center, Milan, Italy; Milena A. De Riz: ${ }^{1}$ Fondazione IRCCS Ca' Granda Ospedale Maggiore Polilclinico, Via F. Sforza 35, 20122 Milan, Italy, ${ }^{2}$ University of Milan, Dino Ferrari Center, Milan, Italy; Alessio Di Fonzo: ${ }^{1}$ Fondazione IRCCS Ca' Granda Ospedale Maggiore Polilclinico, Via F. Sforza 35, 20122 Milan, Italy, ${ }^{2}$ University of Milan, Dino Ferrari Center, Milan, Italy; Giulia Franco: ${ }^{1}$ Fondazione IRCCS Ca' Granda Ospedale Maggiore Polilclinico, Via F. Sforza 35, 20122 Milan, Italy, ${ }^{2}$ University of Milan, Dino Ferrari Center, Milan, Italy; Giorgio G. Fumagalli: ${ }^{1}$ Fondazione IRCCS Ca' Granda Ospedale Maggiore Polilclinico, Via F. Sforza 35, 20122 Milan, Italy, ${ }^{2}$ University of Milan, Dino Ferrari Center, Milan, Italy; Isabella Ghione: ${ }^{1}$ Fondazione IRCCS Ca' Granda Ospedale Maggiore Polilclinico, Via F. Sforza 35, 20122 Milan, Italy, ${ }^{2}$ University of Milan, Dino Ferrari Center, Milan, Italy; Francesca Magri: ${ }^{1}$ Fondazione IRCCS Ca' Granda Ospedale Maggiore Polilclinico, Via F. Sforza 35, 20122 Milan, Italy, ${ }^{2}$ University of Milan, Dino Ferrari Center, Milan, Italy; Filippo Martinelli Boneschi: ${ }^{1}$ Fondazione IRCCS Ca' Granda Ospedale Maggiore Polilclinico, Via F. Sforza 35, 20122 Milan, Italy, ${ }^{2}$ University of Milan, Dino Ferrari Center, Milan, Italy; Megi Meneri: ${ }^{1}$ Fondazione IRCCS Ca' Granda Ospedale Maggiore Polilclinico, Via F. Sforza 35, 20122 Milan, Italy, ${ }^{2}$ University of Milan, Dino Ferrari Center, Milan, Italy; Edoardo Monfrini: ${ }^{1}$ Fondazione IRCCS Ca' Granda Ospedale Maggiore Polilclinico, Via F. Sforza 35, 20122 Milan, Italy, ${ }^{2}$ University of Milan, Dino Ferrari Center, Milan, Italy; Monica Sciacco: ${ }^{1}$ Fondazione IRCCS Ca' Granda Ospedale Maggiore Polilclinico, Via F. Sforza 35, 20122 Milan, Italy, ${ }^{2}$ University of Milan, Dino Ferrari Center, Milan, Italy; Maria Cristina Saetti: ${ }^{1}$ Fondazione IRCCS Ca' Granda Ospedale Maggiore Polilclinico, Via F. Sforza 35, 20122 Milan, Italy, ${ }^{2}$ University of Milan, Dino Ferrari Center, Milan, Italy; Daniele Velardo: ${ }^{1}$ Fondazione IRCCS Ca' Granda Ospedale Maggiore Polilclinico, Via F. Sforza 35, 20122 Milan, Italy, ${ }^{2}$ University of Milan, Dino Ferrari Center, Milan, Italy.

Funding This research was supported by the Italian Ministry of Health (Ricerca Corrente).

Data availability and code availability Not applicable.

\section{Compliance with ethical standards}

Conflict of interest The authors declare that they have no conflict of interest.

Ethics approval The study was approved by the local Ethics Committee of the Fondazione IRCCS Ca' Granda Ospedale Maggiore Policlinico (363_2020, approved 05.05.2020).

Statement of human rights All procedures performed in studies involving human participants were in accordance with the ethical standards of the institutional and/or national research committee and with the 1964 Helsinki declaration and its later amendments or comparable ethical standards.
Statement of the welfare of animals This article does not contain any studies with animals performed by any of the authors.

Informed consent Informed consent was obtained from all individual participants included in the study.

\section{References}

1. Protezione Civile: Coronavirus. http://www.protezionecivile.gov. it/attivita-rischi/rischiosanitario/ emergenze/coronavirus.

2. Zehender G, Lai A, Bergna A, Meroni L, Riva A, Balotta C et al (2020) Genomic characterization and phylogenetic analysis of SARS-COV-2 in Italy. J Med Virol 92(9):1637-1640

3. Amendola A, Bianchi S, Gori M, Colzani D, Canuti M, Borghi E et al (2020) Evidence of SARS-CoV-2 RNA in an oropharyngeal swab specimen, Milan, Italy, early December 2019. Emerg Infect Dis $27(2)$

4. Grasselli G, Pesenti A, Cecconi M (2020) Critical care utilization for the COVID-19 outbreak in Lombardy, Italy: early experience and forecast during an emergency response. JAMA 19:2-3

5. Zhou F, Yu T, Du R, Fan G, Liu Y, Liu Z et al (2020) Clinical course and risk factors for mortality of adult inpatients with COVID-19 in Wuhan, China: a retrospective cohort study. Lancet 395:1054-1062

6. Shi H, Han X, Jiang N, Cao Y, Alwalid O, Gu J et al (2020) Radiological findings from 81 patients with COVID-19 pneumonia in Wuhan, China: a descriptive study. Lancet Infect Dis 20(4):425-434

7. Bikdeli B, Madhavan MV, Jimenez D, Chuich T, Dreyfus I, Driggin E et al (2020) COVID-19 and thrombotic or thromboembolic disease: implications for prevention antithrombotic therapy, and follow-up. J Am CollCardiol 75(23):2950-2973

8. Connors JM, Levy JH (2020) COVID-19 and its implications for thrombosis and anticoagulation. Blood 135(23):2033-2040

9. Mao L, Jin H, Wang M, Hu Y, Chen S, He Q et al (2020) Neurologic manifestations of hospitalized patients with coronavirus disease 2019 in Wuhan, China. JAMA Neurol 77(6):1-9

10. Zubair AS, McAlpine LS, Gardin T, Farhadian S, Kuruvilla DE, Spudich S (2020) Neuropathogenesis and neurologic manifestations of the coronaviruses in the age of coronavirus disease 2019: a review. JAMA Neurol 77(8):1018-1027

11. Adams HP Jr, Bendixen BH, Kappelle LJ, Biller J, Love BB, Gordon DL et al (1993) Classification of subtype of acute ischemic stroke. Definitions for use in a multicenter clinical trial. TOAST. Trial of Org 10172 in acute stroke treatment. Stroke 24(1):35-41

12. Yaghi S, Bernstein RA, Passman R, Okin PM, Furie KL (2017) Cryptogenic stroke: research and practice. Circ Res 120(3):527-540

13 Varga Z, Flammer AJ, Steiger P, Haberecker M, Andermatt R, Zinkernagel AS et al (2020) Endothelial cell infection and endotheliitis in COVID-19. Lancet 395(10234):1417-1418

14. Barton LM, Duval EJ, Stroberg E, Ghosh S, Mukhopadhyay S (2020) COVID-19 Autopsies, Oklahoma, USA. Am J of Clin Pathol 153(6):725-733

15. Avula A, Nalleballe K, Narula N, Sapozhnikov S, Dandu V, Toom $S$ et al (2020) COVID-19 presenting as stroke. Brain Behav Immun. 87:115-119

Publisher's Note Springer Nature remains neutral with regard to jurisdictional claims in published maps and institutional affiliations. 